\title{
Dependence of the defect introduction rate on the dose of irradiation of p-Si by fast-pile neutrons
}

\author{
A.P. Dolgolenko, M.D. Varentsov, G.P. Gaidar* , P.G. Litovchenko \\ Institute for Nuclear Research, NAS of Ukraine, \\ 47, prospect Nauky, 03680 Kyiv, Ukraine, fax: 380-44-5254463 \\ *Corresponding author:e-mail: gaidar@kinr.kiev.ua
}

\begin{abstract}
We have studied the high-resistance samples of $\mathrm{p}$-Si $\left(p_{00}=(3.3 \pm 0.5) \times\right.$ $\left.\times 10^{12} \mathrm{~cm}^{-3}\right)$ and $n$-Si $\left(n_{0}=(2.0 \pm 0.3) \times 10^{12} \mathrm{~cm}^{-3}\right)$ grown by the floating-zone technique after the irradiation by fast-pile neutrons at $287 \mathrm{~K}$. The dose and the temperature dependences of the effective concentration of carriers have been measured. The calculation has been carried out in the framework of Gossick's corrected model. It is shown that the radiation hardness of $n$ - and $p$ - $\mathrm{Si}$, on the one hand, is defined by clusters, and, on the other hand, by vacancy defects (acceptors) in $n$-Si and by interstitial defects (donors and acceptors) in $p$-Si. We have determined that, during the irradiation of $p$-Si by small doses of neutrons, the change of a charge state of interstitial defects leads to the annealing of these defects and to a decrease of their introduction rate.
\end{abstract}

Keywords: silicon, neutron irradiation, radiation hardness, radiation defect, cluster.

Manuscript received 19.10.07; accepted for publication 19.12.07; published online 30.01.08.

\section{Introduction}

The radiation hardness of semiconductor devices keeps the attraction of researches. The actuality of the data on the type and the characteristics of radiation defects in such materials increases significantly due to the realization of the project of a Super Large Hadron Collider (SLHC) with a luminosity of $\sim 10^{35} \mathrm{~cm}^{-2} \mathrm{~s}^{-1}$. This demands the detectors with radiation hardness more than $\sim 10^{16} \mathrm{n}^{\mathrm{o}} \mathrm{cm}^{-2}$. The high carrier mobility in $n$-type Si (by 3.5 orders more than that for $p$-type) and its working capacity after $n \rightarrow p$ conversion make the ionizing radiation detectors fabricated on $n$-type $\mathrm{Si}$ very attractive.

It should be noted that the formation of defects substantially depends on the temperature, at which the samples are irradiated. For example, the irradiation below $120 \mathrm{~K}$ decreases the defect introduction rate by several orders [1]. In [2], it was shown that the intensity of near-edge absorption and the rate of post irradiation annealing of defect clusters are considerably higher for silicon crystals irradiated by fast neutrons at $100 \mathrm{~K}$. This can be explained by the high introduction rate of non reorientation divacancies, which are strongly annealed under the temperature higher than $140 \mathrm{~K}$. The lowtemperature irradiation also suppresses the participation of oxygen in the formation of electrically active oxygencontained defects.

Taking into account the above-mentioned, the main objectives of the present paper are: (i) to measure and to describe the temperature dependence of the hole effecttive concentration in $p$-Si irradiated by various fluences of fast neutrons; (ii) to compare the radiation hardness of $n$ - and $p$-Si; (iii) to calculate the dependence of the defect introduction rate on the irradiation dose.

\section{Experiment}

High-resistance samples of $p$-Si $\left(p_{00}=(3.3 \pm 0.5) \times\right.$ $\left.\times 10^{12} \mathrm{~cm}^{-3}\right)$ and $n$-Si $\left(n_{0}=(2.0 \pm 0.3) \times 10^{12} \mathrm{~cm}^{-3}\right)$ grown by the floating-zone technique with a specific resistance of near $10 \mathrm{kOhm} \cdot \mathrm{cm}$ were irradiated by various fluences of fast-pile neutrons at a temperature of $287 \mathrm{~K}$. The irradiation was performed in the horizontal channel of a water-moderated research reactor (WWR-M) with a neutron beam intensity of $5 \times 10^{8} \mathrm{n}^{\circ} \mathrm{cm}^{-2} \mathrm{~s}^{-1}$ and the fluences from $10^{11}$ till $2 \times 10^{14} \mathrm{n}^{\circ} \mathrm{cm}^{-2}$. The neutron fluence was determined by a ${ }^{32} \mathrm{~S}$ threshold detector with accuracy of $10 \%$ for the energy of neutrons starting from $\sim 100 \mathrm{keV}$. The measurements of conductivity and the Hall coefficient were carried out by the van der Pauw compensatory 
method on samples of $10 \times 10 \times 1 \mathrm{~mm}$ in size with accuracy of $3 \%$. Contacts were formed by the rubbing of aluminum into the silicon polished surface.

\section{Results}

The temperature dependences of the effective hole concentration for $p$-Si irradiated by different fluences of fast-pile neutrons are presented in Figs. 1 and 2. The evaluated values for the parameters of radiation defects are presented in Table 1.

Fig. 3 shows the dependences of the effective concentrations of carriers in $n$ - and $p$-type $\mathrm{Si}$ on the fluence of fast-pile neutrons.

The dependences of the introduction rates of radiation defects on the irradiation time (at $287 \mathrm{~K}$ ) are presented in Fig. 4.

Table 1. Radiation defect parameters for $p$-Si irradiated by fast-pile neutrons at $287 \mathrm{~K}$.

\begin{tabular}{|c|c|c|c|c|c|}
\hline $\begin{array}{c}\Phi, \\
\mathrm{n}^{\mathrm{o}} \mathrm{cm}^{-2}\end{array}$ & $\begin{array}{l}p_{00} \\
\mathrm{~cm}^{-3}\end{array}$ & $N_{a i}, \mathrm{~cm}^{-3}$ & $v_{i}, \mathrm{~cm}^{-1}$ & $\begin{array}{c}E_{v}+E_{a i}, \\
\mathrm{eV}\end{array}$ & $R_{1}, \AA$ \\
\hline $5 \times 10^{12}$ & $\begin{array}{c}2.96 \times \\
\times 10^{12}\end{array}$ & $\begin{array}{l}1.5 \times 10^{13} \\
1.5 \times 10^{12} \\
8.0 \times 10^{11}\end{array}$ & $\begin{array}{c}3 \\
0.3 \\
0.16 \\
\end{array}$ & $\begin{array}{l}0.42 \\
0.51 \\
0.45 \\
\end{array}$ & 36 \\
\hline $\begin{array}{c}7.55 \times \\
\times 10^{12}\end{array}$ & $\begin{array}{l}3.22 \times \\
\times 10^{12}\end{array}$ & $\begin{array}{l}8.0 \times 10^{12} \\
1.6 \times 10^{12} \\
4.9 \times 10^{11} \\
1.0 \times 10^{12}\end{array}$ & $\begin{array}{c}1.06 \\
0.21 \\
6.5 \times 10^{-2} \\
0.13\end{array}$ & $\begin{array}{l}0.42 \\
0.51 \\
0.33 \\
0.45\end{array}$ & 36 \\
\hline $\begin{array}{l}1.0 \times \\
\times 10^{13}\end{array}$ & $\begin{array}{l}2.68 \times \\
\times 10^{12}\end{array}$ & $\begin{array}{c}6 \times 10^{12} \\
1.7 \times 10^{12} \\
6.2 \times 10^{11} \\
\end{array}$ & $\begin{array}{c}0.6 \\
0.17 \\
6.2 \times 10^{-2} \\
\end{array}$ & $\begin{array}{l}0.42 \\
0.51 \\
0.45 \\
\end{array}$ & 36 \\
\hline $\begin{array}{c}1.89 \times \\
\times 10^{13}\end{array}$ & $\begin{array}{l}3.16 \times \\
\times 10^{12}\end{array}$ & $\begin{array}{c}1.7 \times 10^{12} \\
1.16 \times 10^{12} \\
8.5 \times 10^{11} \\
\end{array}$ & $\begin{array}{c}9 \times 10^{-2} \\
6.2 \times 10^{-2} \\
4.5 \times 10^{-2} \\
\end{array}$ & $\begin{array}{l}0.42 \\
0.51 \\
0.45 \\
\end{array}$ & 36 \\
\hline \begin{tabular}{|c|}
$2.26 \times$ \\
$\times 10^{13}$ \\
\end{tabular} & $\begin{array}{l}2.84 \times \\
\times 10^{12} \\
\end{array}$ & \begin{tabular}{|c|}
$2.2 \times 10^{12}$ \\
$1.04 \times 10^{12}$
\end{tabular} & $\begin{array}{l}9.7 \times 10^{-2} \\
4.6 \times 10^{-2}\end{array}$ & $\begin{array}{l}0.42 \\
0.51\end{array}$ & 36 \\
\hline \begin{tabular}{|c|}
$2.64 \times$ \\
$\times 10^{13}$ \\
\end{tabular} & $\begin{array}{r}3.18 \times \\
\times 10^{12}\end{array}$ & $\begin{array}{l}2.5 \times 10^{12} \\
1.3 \times 10^{12}\end{array}$ & $\begin{array}{l}9.5 \times 10^{-2} \\
4.9 \times 10^{-2}\end{array}$ & $\begin{array}{l}0.42 \\
0.51\end{array}$ & 36 \\
\hline \begin{tabular}{|c|}
$2.83 \times$ \\
$\times 10^{13}$ \\
\end{tabular} & $\begin{array}{l}3.17 \times \\
\times 10^{12} \\
\end{array}$ & $\begin{array}{l}2.15 \times 10^{12} \\
1.13 \times 10^{12} \\
\end{array}$ & $\begin{array}{c}7.6 \times 10^{-2} \\
4 \times 10^{-2} \\
\end{array}$ & $\begin{array}{l}0.42 \\
0.51 \\
\end{array}$ & 36 \\
\hline \begin{tabular}{|c|}
$3.02 \times$ \\
$\times 10^{13}$ \\
\end{tabular} & $\begin{array}{l}2.97 \times \\
\times 10^{12}\end{array}$ & $\begin{array}{c}2.1 \times 10^{12} \\
7.25 \times 10^{11}\end{array}$ & $\begin{array}{c}7 \times 10^{-2} \\
2.4 \times 10^{-2} \\
\end{array}$ & $\begin{array}{l}0.42 \\
0.51\end{array}$ & 36 \\
\hline \begin{tabular}{|c|}
$3.21 \times$ \\
$\times 10^{13}$ \\
\end{tabular} & $\begin{array}{l}3.18 \times \\
\times 10^{12} \\
\end{array}$ & $\begin{array}{l}2.5 \times 10^{12} \\
6.3 \times 10^{11} \\
\end{array}$ & $\begin{array}{c}7.8 \times 10^{-2} \\
2 \times 10^{-2}\end{array}$ & $\begin{array}{l}0.42 \\
0.51\end{array}$ & 36 \\
\hline \multicolumn{6}{|c|}{ Annealing at $292 \mathrm{~K} ; 8.04 \times 10^{6} \mathrm{~s}$} \\
\hline $5 \times 10^{12}$ & $\begin{array}{r}2.96 \times \\
\times 10^{12}\end{array}$ & $\begin{array}{c}1.0 \times 10^{11} \\
1.25 \times 10^{12} \\
1.6 \times 10^{11} \\
2.0 \times 10^{11} \\
\end{array}$ & \begin{tabular}{|c}
$2 \times 10^{-2}$ \\
$2.5 \times 10^{-2}$ \\
$3.2 \times 10^{-2}$ \\
0.1
\end{tabular} & $\begin{array}{l}0.42 \\
0.36 \\
0.33 \\
0.26 \\
\end{array}$ & 20 \\
\hline
\end{tabular}

Note. The level with energy $E_{\mathrm{v}}+0.42 \mathrm{eV}$ is the acceptor level and all other levels with energy $E_{\mathrm{v}}+E_{a i}$ are the donor ones. $\Phi$ is a fluence of fast-pile neutrons, $p_{00}$ is a carrier concentration before the irradiation; $N_{a i}$ is the concentration of the $i$-th defect with energy level in the forbidden band $E_{\mathrm{v}}+E_{a i} ; v_{i}$ is the introduction rate of the $i$-th defect; and $R_{1}$ is the average radius of the clusters of defects.

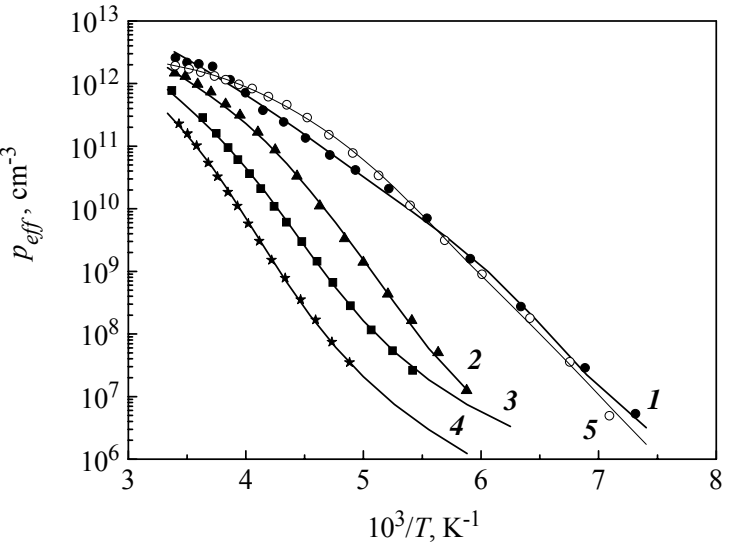

Fig. 1. Temperature dependences of the effective carrier concentration $\left(p_{\text {eff }}\right)$ for $p$-Si irradiated by fast-pile neutrons with fluences: $1-5 \times 10^{12} ; 2-7.55 \times 10^{12} ; 3-1.0 \times 10^{13} ; 4$ $1.89 \times 10^{13} \mathrm{n}^{\circ} \mathrm{cm}^{-2} ; 5-$ after annealing $\left(8.04 \times 10^{6} \mathrm{~s}\right)$ under a fluence of $5 \times 10^{12} \mathrm{n}^{\circ} \mathrm{cm}^{-2}$ at $292 \mathrm{~K}$. Symbols present the experimental data; solid lines are the results of calculations.

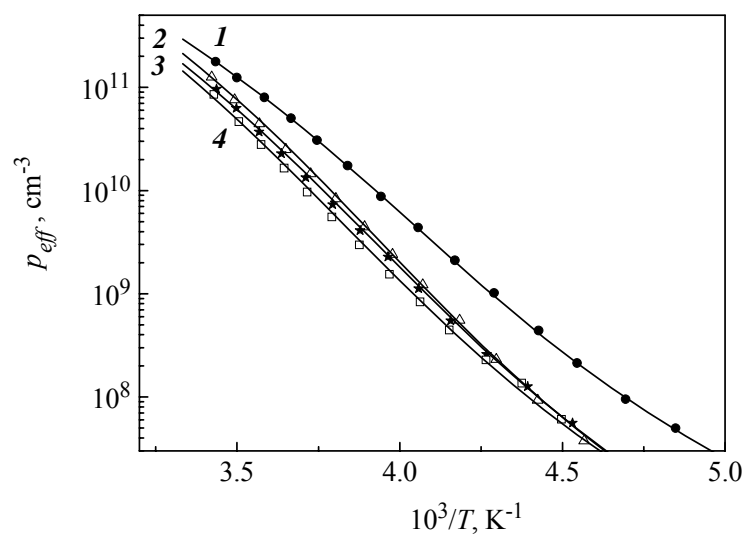

Fig. 2. Temperature dependences of effective carrier concentration $\left(p_{\text {eff }}\right)$ for $p$-Si irradiated by fast-pile neutrons with fluences: $1-2.264 \times 10^{13} ; 2-2.64 \times 10^{13} ; 3$ $2.83 \times 10^{13} ; 4-3.02 \times 10^{13} \mathrm{n}^{\circ} \mathrm{cm}^{-2}$. Symbols present the experimental data; solid lines are the results of calculations.

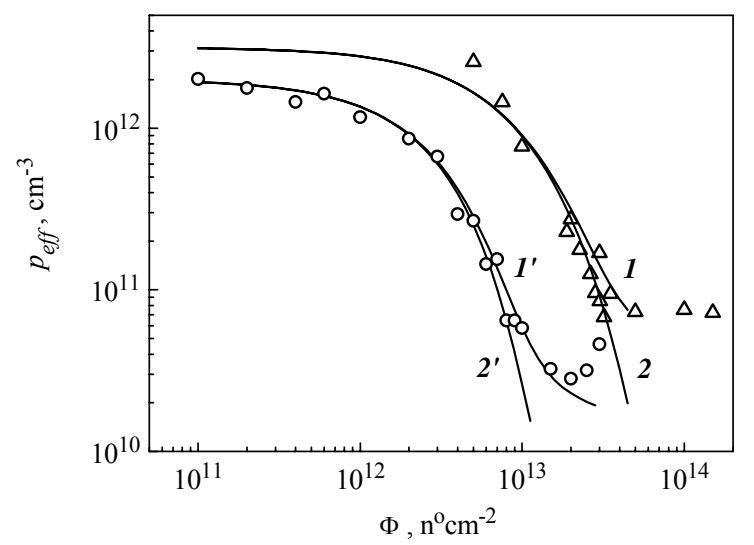

Fig. 3. Dependence of the effective concentrations of carriers $\left(p_{\text {eff }}\right)$ on the fluence $(\Phi)$ of fast-pile neutrons at $292 \mathrm{~K}$ in silicon samples, grown by the floating-zone technique: $\Delta-p$ Si $\left(p_{00}=3.2 \times 10^{12} \mathrm{~cm}^{-3}\right)$ and $\circ-n$-Si $\left(n_{0}=2.0 \times 10^{12} \mathrm{~cm}^{-3}\right)$; the results of calculations with $\left(1, l^{\prime}\right)$ and without $\left(2,2^{\prime}\right)$ account of the additional overlapping of defect clusters are presented by solid lines. 


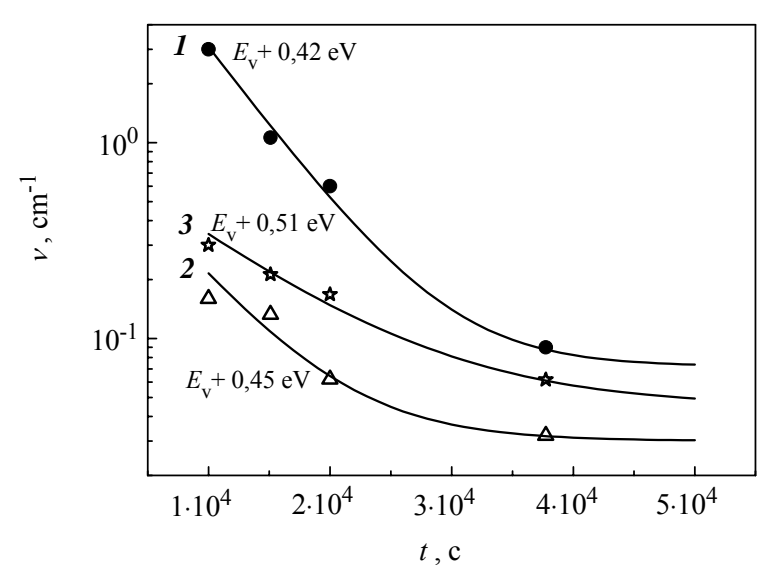

Fig. 4. Dependence of the introduction rate of defects with energy levels $E_{v}+0.42 \mathrm{eV} \quad(1), \quad E_{v}+0.45 \mathrm{eV}$ (2), and $E_{v}+0.51 \mathrm{eV}(3)$ on the irradiation time of $p$-Si samples at $287 \mathrm{~K}$.

\section{Calculation of the temperature and dose dependences of the effective carrier concentration}

The primary knock-on silicon atoms, originated due to the irradiation of Si by fast-pile neutrons, produce the vacancies and interstitial defects along trajectories of motion. At the end of a trajectory, the high local concentration of defects is created (after their athermic realignment), which leads to the formation of the clusters of vacancy- and also interstitial-type defects in the conductive matrix of a sample.

The process of accumulation of the clusters of defects in a separate volume is similar to the activation and decay of radioactive nuclei. Thus, the volume fraction $(f)$, occupied by clusters (due to the introduction of point defects), with regard for the overlapping of clusters in accordance with [3], can be evaluated as

$f=(1-\exp (-\Sigma V \Phi)) \cdot \exp \left(-\Sigma_{1} V \Phi\right)$

where $\Sigma=0.15 \mathrm{~cm}^{-1}$ is the macroscopic cross-section of the introduction of clusters due to the irradiation by fastpile neutrons; $\Sigma_{1}$ is the probability of the overlapping of the clusters of defects, $\mathrm{cm}^{-1} ; V$ is the separate volume of an average defect cluster, $\mathrm{cm}^{3} ; \Phi$ is the fluence of fastpile neutrons, $\mathrm{n}^{\mathrm{o}} \mathrm{cm}^{-2}$.

The probability of the overlapping of the clusters of defects is substantially smaller than that of the formation of the clusters of defects: $\Sigma_{1}<<\Sigma$.

Within Gossick's model for the cluster volume [4], the effective concentration of carriers depending on the temperature and a small fluence is given by the formula [5]

$$
\begin{aligned}
& p_{\text {eff }}(T, \Phi)=p(T, \Phi) \times \\
& \times \exp \left[-\frac{4 \pi \varepsilon \varepsilon_{0} \sum R_{1} \Phi}{P_{2}(T, \Phi) q^{2}}\left(\mu_{p}-k T \ln \frac{N_{V}(T)}{P_{2}(T, \Phi)}\right)\right],
\end{aligned}
$$

where $p(T, \Phi)$ is the concentration of carriers in the $p$-Si conduction matrix; $P_{2}(T, \Phi)$ is the concentration of screening centers in the space charge region of defect clusters; $N_{V}(T)$ is the effective density of states in the valence zone of $p$ - $\mathrm{Si} ; R_{1}$ is the average radius of defects gathering regions; $\varepsilon$ and $\varepsilon_{0}$ are the dielectric constants of a material and vacuum, respectively; $q$ is the carrier charge; $\mu_{p}$ is the Fermi level position relative to the top of the valence zone at the center of the damage region of a defect cluster.

For the correct calculation of $p_{\text {eff }}(T, \Phi)$, the compensation of the conductive matrix must be taken into account, which leads to the additional overlapping in the space charge region of defect clusters in accordance with (1).

The Fermi level position is determined by thermodynamical characteristics of the system. Thus, $\mu$ and $k T \ln \left(N_{c}(T) / N_{2}(T, \Phi)\right)$ for $n$-Si can be defined as an increment of the free energy of the system (a cluster and the conducting matrix) on the addition of one electron at the constant volume and temperature. Then $q^{2} N_{2}(T, \Phi) / 4 \pi \varepsilon \varepsilon_{0} \Sigma R_{1} \Phi$ can be described as a decrease in the free energy for the full system on the formation of $\Sigma \Phi$ defect clusters per volume unit. If the energy direction should be changed, the above-mentioned considerations will correspond to holes in $p$-Si.

Divacancies are multi-charge centers, whose donor $\left(E_{v}+0.25 \mathrm{eV}\right)$ and acceptor $\left(E_{c}-0.42 \mathrm{eV}\right)$ levels determine the position of the Fermi level $\mu=$ $E_{v}+0.475 \mathrm{eV}$ in the defect clusters formed by fast neutrons in $p$-Si. In the intrinsic silicon, the Fermi level should be located at the level of a neutral divacancy which becomes apparent in experiments as the recombination level $E_{c}-0.62 \mathrm{eV}$ under the full overlapping of defect clusters. With decrease in temperature, the electrons and holes are captured on the level $E_{v}+0.52 \mathrm{eV}$ and recombine with one another. The Fermi level moves to the middle of the forbidden band. At the capture of a free electron (supplied by the ionization of dopants), the energy of a divacancy will increase by $0.165 \mathrm{eV}$. Thus, with increase in the $n-\mathrm{Si}$ doping level $\left(n_{0}\right)$, the Fermi level in a cluster can be defined as

$$
\mu=E_{\mathrm{c}}-0.6+0.033 \log n_{0} / n_{i},
$$

where $n_{0}$ is the concentration of electrons in the conduction band before the irradiation, $\mathrm{cm}^{-3}$; $n_{i}=10^{10} \mathrm{~cm}^{-3}$ is the concentration of carriers in intrinsic silicon. Eq. (3) corresponds to the experimentally determined position of the Fermi level in $n$-Si (with different $n_{0}$ ) irradiated by the fluence of fast-pile neutrons, at which the defect clusters are fully overlapped.

Let us consider $p$-Si doped by boron with non compensated concentration $N_{a}$ in the range from room temperature to liquid nitrogen one. Let fast-pile neutrons 
homogenously form the donor-type point defects (apart from the defect gathering region) with concentration $N_{d}<N_{a}$ and the acceptor-type defects. We consider $p$-Si as non-degenerated $\left(N_{a}<10^{14} \mathrm{~cm}^{-3}\right)$. Then, as the temperature of a $p$-Si sample increases from $77 \mathrm{~K}$, we obtain some concentration of holes (in the valence band) due to the thermal excitation of holes from the $E_{d}$ level both in the conduction matrix $p_{1}(T, \Phi)$ and in the spacecharge regions of defect clusters $P_{3}(T, \Phi)$

$$
\begin{aligned}
& p_{1}\left(T, \Phi, E_{d}\right)=\frac{1}{2}\left(N_{a}-\frac{N_{d}(\Phi)}{\lambda}-p_{11}\left(E_{d}\right)\right) \times \\
& \times\left(\sqrt{1+\frac{4 N_{a} p_{11}\left(E_{d}\right)}{\left(N_{a}-\frac{N_{d}(\Phi)}{\lambda}-p_{11}\left(E_{d}\right)\right)^{2}}}+1\right), \\
& p_{11}\left(E_{d}\right)=g N_{V}(T) \exp \left(-\frac{E_{d}}{\lambda k T}\right),
\end{aligned}
$$

where $g=2$ is the factor of donor level degeneration in $p$ - $\mathrm{Si} ; N_{d}(\Phi)$ is the concentration of radiation-introduced donor defects after the irradiation by the fluence $\Phi$; $p_{11}\left(E_{d}\right)$ is the concentration of holes in the valence band of $p$-Si under condition that the Fermi level coincides with the $E_{d}$ level in the conductive matrix or the effective level $E_{d} / \lambda$ in the space-charge regions of defect clusters. Equation (4) was obtained from solving the quadratic expression which follows from the condition of electroneutrality [5].

In a similar manner with increase in the temperature of a $p$-Si sample from $77 \mathrm{~K}$, we obtain some concentration of holes in the valence band due to the thermal excitation of carriers from the acceptor level $E_{a}$ both in the conducting matrix $p_{0}(T, \Phi)$ and in the spacecharge regions of defect clusters $P_{4}(T, \Phi)$

$$
\begin{aligned}
& p_{0}\left(T, \Phi, E_{a}\right)=\frac{1}{2} p_{11}\left(E_{a}\right)\left(\sqrt{1+\frac{4 N_{a}(\Phi)}{p_{11}\left(E_{a}\right)}}-1\right), \\
& p_{11}\left(E_{a}\right)=g_{1} N_{V}(T) \exp \left(-\frac{E_{a}}{\lambda k T}\right),
\end{aligned}
$$

where $g_{1}=0.5$ is the degree of acceptor level degeneration in $p$-Si; and $N_{a}(\Phi)$ is the concentration of radiation-introduced acceptor-type defects after the irradiation by the fluence $\Phi$.

If the donor and acceptor defects are located in the conducting matrix of $p$-Si, then $\lambda=1$. But if they are in the space-charge regions of defect clusters, then $\lambda=1.2$. The analysis of the temperature dependences $p_{\text {eff }}(T, \Phi)$ shows that their better description will be obtained on the supposition that defects (with effective level $E_{a} / \lambda$ ) are present in the space-charge regions, which compensates the screening effect of a shallow dopant (boron). The energy position of the mentioned level in the conducting matrix in $p$-Si is $E_{a}$. Then the additional hole concentration in the valence band of the conducting matrix of samples of $p$-Si is equal to $p=p_{0}+p_{1}$, and the supplementary concentration of screening centers in the space-charge regions of defect clusters is $P_{2}=P_{4}+P_{3}$.

Figures 1 and 2 show the calculated [according to Eqs. (2), (4), and (5)] the temperature dependences of effective hole concentrations in the valence band of $p-\mathrm{Si}$ under irradiation by various fluences of fast-pile neutrons. The parameters of calculations are presented in Table 1. It was supposed that, in the case of the absence of a statistical interaction between the levels of radiation defects, the carrier concentration in the conducting matrix of $p$-Si can be determined by the calculation of the total concentration of holes $\sum_{i} p_{i}\left(T, \Phi, E_{i}\right)$, which will be supplied into the valence band due to the ionization of acceptor and donor levels:

$p(T, \Phi)=\sum_{i} p_{i}\left(T, \Phi, E_{i}\right)-p_{00}+N_{D}(\Phi)$.

Here, $i$ is the index running from 1 to 3 (we assume the presence of one acceptor and two donor defect levels in the conductive matrix); $p_{00}$ is the hole concentration in $p$-Si before irradiation; and $N_{D}(\Phi)$ is the concentration of the deepest donor levels.

The concentration of screening centers in the space-charge regions of defect clusters can be determined with accordance to (4) and (5) for $\lambda=1.2$ as

$P_{2}(T, \Phi)=\sum_{i} P_{2 i}\left(T, \Phi, E_{i}\right)-p_{00}+\frac{N_{D}(\Phi)}{\lambda}$,

where $i$ is the index running from 1 to 3 (we assume the presence of one acceptor and two donor defect levels in the space-charge regions); and $N_{D}(\Phi)$ is the concentration of radiation-induced defects.

\section{Discussion}

The analysis of the literature data shows a lot of data concerning the radiation defects, whose energy levels are located in the forbidden band with a spacing from 0.02 to $0.05 \mathrm{eV}$ [6]. Nevertheless, many defect levels are undefined (see, e.g., [7]). However, the problem of radiation defect levels is complicated by the fact that the theoretical calculation of the energy level position for deep states is unsolved so far despite some successful results.

In [8], the original scheme of energy levels for intrinsic radiation defects, which is based both on the literature data and the original experiment, was proposed. It takes the following positions into account: (i) radiation defects form the additional energy levels in the forbidden band, and the intrinsic defects in silicon are amphoteric; (ii) under capture of one or two electrons on acceptor levels of a divacancy or a di- 
interstitial, the position of levels in the forbidden gap of silicon is changed by the value $\Delta E_{0}=0.165 \pm 0.005 \mathrm{eV}$, and, in the case of vacancies or interstitials, the mentioned value is added twice; (iii) the modification of a divacancy by carbon increases the energy position of acceptor levels by the value $\Delta E_{1}=0.035 \mathrm{eV}$ and decreases the energy of donor levels, whereas the addition of oxygen to a divacancy decreases the acceptor level energy and increases the donor level energy of a divacancy by the value $\Delta E_{2}=0.06 \mathrm{eV}$.

The analysis and the results presented in [8] gave us a possibility to determined the fact that the energy of acceptor levels is increased by value $\Delta E=0.33 / \xi$ under the electron capture, where $\xi$ is the number of vacancies in the multivacancy defects $(1 \leq \xi \leq 5)$.

The positions of acceptor levels relative to the bottom of the conduction band and those of donor levels relative to the valence band top for intrinsic radiation defects in $p$-Si are presented in Table 2.

We assume (see the Table in [8]) that $E_{\mathrm{v}}+0.42 \mathrm{eV}$ belongs to the acceptor level of interstitial levels $\left(\mathrm{I}^{-/ 0}\right)$, and $E_{\mathrm{v}}+0.45 \mathrm{eV}$ belongs to the donor level of diinterstitials $\left(\mathrm{I}_{2}{ }^{0 /+}\right)$. The increase in the number of negatively polarized oxygen neighbors in the series of $\mathrm{C}_{i}, \mathrm{C}_{i} \mathrm{O}_{i}, \mathrm{C}_{i} \mathrm{O}_{2 i}$ leads to an upward shift of their donor levels: $\quad E_{v}+0.28 \mathrm{eV}, \quad E_{v}+0.34 \mathrm{eV}, \quad E_{v}+0.39 \mathrm{eV}$, respectively. This can be explained by the effect of increasing the repulsive electrostatic potential that originates from oxygen atoms [9]. Thus, the observed level $E_{v}+0.51 \mathrm{eV}$ can be attributed to $\mathrm{I}_{2} \mathrm{O}_{i}^{0 /+}$ defects.

In Fig. 3 , the results of theoretical calculations of the dependence of the effective carrier concentration on the fluence of fast-pile neutrons for $n$ - and $p$-Si with and without additional overlapping of defect clusters, according to (1), are presented by solid lines. The calculations were carried out in the frame of Gossick's corrected model for the defect clusters with average radii of defect gathering regions $R_{n}=40 \AA$ and $R_{p}=36 \AA$ for the samples of $n$ - and $p$-type silicon, respectively. While calculating the carrier concentration in the conducting matrix by (4) and (5), it was usually supposed that $N_{a}=p_{00}, N_{a}(\Phi)=v_{a} \times \Phi$, and $N_{d}(\Phi)=v_{d} \times \Phi$, where $v_{d}$ is the rate of hole removal by donor defects, and $v_{a}$ is the rate of free hole generation by deep acceptor defects. In the description of the dose dependence of the carrier

Table 2. Radiation annealing of interstitial-type defects.

\begin{tabular}{|c|c|c|c|c|c|}
\hline $\begin{array}{c}\text { De- } \\
\text { fect }\end{array}$ & $\begin{array}{c}T_{\text {anneal }}, \\
\mathrm{K}\end{array}$ & $\begin{array}{c}\text { Activa- } \\
\text { tion } \\
\text { energy, } \\
\mathrm{eV}\end{array}$ & $\begin{array}{c}\text { Frequen- } \\
\text { cy } \\
\text { factor, } \\
\mathrm{s}^{-1}\end{array}$ & $\begin{array}{c}\text { Annealing } \\
\text { reaction }\end{array}$ & $\begin{array}{c}\text { Level } \\
\text { energy, } \\
\mathrm{eV}\end{array}$ \\
\hline $\mathrm{I}^{=/-}$ & 287 & 0.4 & $2.0 \times 10^{3}$ & $\mathrm{I}^{-} \rightarrow \mathrm{I}^{-}$ & $E_{\mathrm{V}}+0.42$ \\
\hline $\mathrm{I}_{2}{ }^{0 /+}$ & 287 & 0.42 & $4.0 \times 10^{3}$ & $\mathrm{I}_{2}{ }^{+} \rightarrow \mathrm{I}_{2}{ }^{0}$ & $E_{\mathrm{v}}+0.45$ \\
\hline $\mathrm{I}_{2} \mathrm{O}_{\mathrm{i}}^{0 /+}$ & 287 & 0.42 & $2.5 \times 10^{3}$ & $\begin{array}{c}\mathrm{I}_{2} \mathrm{O}_{\mathrm{i}}{ }^{+} \rightarrow \\
\rightarrow \mathrm{I}_{2} \mathrm{O}_{\mathrm{i}}{ }^{0}\end{array}$ & $E_{\mathrm{v}}+0.51$ \\
\hline
\end{tabular}

concentration, the introduction rate for a donor level $\left(E_{v}+0.51 \mathrm{eV}\right)$ is obtained as $v_{d}=0.06 \mathrm{~cm}^{-1}$, and, for an acceptor level $\left(E_{\mathrm{v}}+0.42 \mathrm{eV}\right)$, the value $v_{a}=0.1 \mathrm{~cm}^{-1}$ was used. For $n-\mathrm{Si}$, the introduction rate of threevacancy acceptor defects $\left(E_{\mathrm{c}}-0.49 \mathrm{eV}\right)$ was taken as $v=0.25 \mathrm{~cm}^{-1}$. It was found that the average value of the introduction rate for defects allows one to get a satisfactory description of the dose dependence $p_{\text {eff }}(\Phi)$. According to (3), the Fermi level position in the clusters formed by fast-pile neutrons in $n-\mathrm{Si}$ is $\mu=$ $E_{\mathrm{c}}-0.524 \mathrm{eV}$. Fig. 3 shows that, after the irradiation with a dose of $\sim 5 \times 10^{13} \mathrm{n}^{\mathrm{o}} \mathrm{cm}^{-2}$, the carrier concentration in $p$-Si reaches a constant value of $8 \times 10^{10} \mathrm{~cm}^{-3}$, and, consequently, with the defect cluster overlapping, the Fermi level stands near $E_{v}+0.476 \mathrm{eV}$ (in the $p$-Si forbidden gap), which is still unchangeable in the wide range of the doping levels of samples. The calculation shows that the probability of the additional overlapping in the space-charge region of defect clusters in $n$-Si is $\Sigma_{1}=0.006 \mathrm{~cm}^{-1}$, whereas $\Sigma_{1}=0.001 \mathrm{~cm}^{-1}$ for $p$-Si.

The radiation hardness for $n$-Si can be confidently determined as $R_{h}=n_{0} / v$, where $v$ is the removal rate of electrons in the conducting matrix. Then, for $p-\mathrm{Si}$, it is

$R_{h}=\frac{p_{0}+v_{a}^{*} \Phi-v_{d}^{*} \Phi}{v_{d}^{*}}=\frac{p_{0}}{2 v_{d}^{*}-v_{a}^{*}}$,

where $v_{a}^{*}=0.08 \mathrm{~cm}^{-1}$, and $v_{d}^{*}=0.06 \mathrm{~cm}^{-1}$ are, respecttively, the rates of introduction and removal of holes at $292 \mathrm{~K}$. Hence, the radiation hardness of $p$-Si is by one order higher than that for $n$-Si irradiated by fast-pile neutrons. The above-determined radiation hardness is, in fact, the dose at which the conductivity of a sample is minimal.

Figure 3 shows that, at low radiation doses, the experimental values of effective carrier concentration don't blend with the overall picture of the $p_{\text {eff }}$ dependence on the fluence of fast-pile neutrons. The defect introduction rates presented in Table 1 (from the calculation of the temperature dependence of $p_{\text {eff }}$ ) are decreased by factors of 3-5 with increase in the irradiation dose in the range from $5 \times 10^{12}$ to $10^{13} \mathrm{n}^{\circ} \mathrm{cm}^{-2}$. The sample storage at room temperature during three months results in that the defect levels $E_{v}+0.51 \mathrm{eV}$ and $E_{v}+0.45 \mathrm{eV}$ are fully annealed, and the introduction rate of $E_{v}+0.42 \mathrm{eV}$ level is considerably decreased. After the irradiation with a dose of near $5 \times 10^{12} \mathrm{n}^{\mathrm{o}} \mathrm{cm}^{-2}$, the Fermi level position into the conductive matrix is $E_{v}+0.38 \mathrm{eV}$. This means that the donor levels of $\mathrm{I}_{2}^{+}$and $\mathrm{I}_{2} \mathrm{O}_{i}^{+}$are in the positively charged state, and interstitial atom (I) with the acceptor level $\left(E_{v}+0.42 \mathrm{eV}\right)$ is, in general, in the neutral state. In [10] with the use of the first-order annealing kinetics, the activation energy $\left(E_{a}=0.6 \pm 0.2 \mathrm{eV}\right)$ and the frequency factor $v \cong 10^{8} \mathrm{~s}^{-1}$ for the isothermal annealing of $\mathrm{I}_{2}^{+}(\mathrm{P} 6)$ (at 370 and $344 \mathrm{~K}$ ) were evaluated. After the irradiation by gamma-rays $\left({ }^{60} \mathrm{Co}\right)$ and by fast-pile neutrons of 
intrinsic $p$-Si, the authors [11] observed the annealing of defects with the energy level $\left(E_{v}+0.40 \mathrm{eV}\right)$ and the activation energy $E_{a}=0.85 \mathrm{eV}$. According to Table 2, the level $\left(E_{v}+0.42 \mathrm{eV}\right)$ is attributed to an acceptor defect. The energy of migration for interstitial atoms in the neutral charged state $\left(\mathrm{I}^{0}\right)$ is $E_{m} \cong 1.5 \mathrm{eV}$; $E_{m} \cong 0.85 \mathrm{eV}$ is the energy of migration for interstitial atoms in the negatively charged state $\left(\mathrm{I}^{-}\right)$, and a twice negatively charged interstitial atom $\left(\mathrm{I}^{\overline{ }}\right)$ has the energy of migration $E_{m} \cong 0.4 \mathrm{eV}$, according to the literature data. Therefore, a decrease in the introduction rate for defects $\mathrm{I}^{0}\left(E_{v}+0.42 \mathrm{eV}\right), \quad \mathrm{I}_{2}^{+}\left(E_{v}+0.45 \mathrm{eV}\right), \quad$ and $\mathrm{I}_{2} \mathrm{O}_{i}^{+}\left(E_{v}+0.51 \mathrm{eV}\right)$ is determined by the radiation annealing under the irradiation by fast-pile neutrons at a temperature of $287 \mathrm{~K}$. The dependence of the defect introduction rate on the time of irradiation by fast-pile neutrons of $p$-Si $\left(\bar{p}_{00}=3.0 \times 10^{12} \mathrm{~cm}^{-3}\right)$ is presented in Fig. 4 . The obtained activation energies for the radiation annealing of the above-mentioned defects and their frequency factors are presented in Table 2.

\section{Conclusions}

We have found that, under the neutron irradiation of $p$-Si at $287 \mathrm{~K}$ (see Table 2), only the changes of charged states of interstitial and di-interstitial atoms lead to a change of their activation energy and the annealing.

It is established that the radiation hardness of $n$ and $p$-type silicon is determined by defect clusters, on the one hand, and vacancy-type defects (acceptors) in n$\mathrm{Si}$ and interstitial-type defects (donors and acceptors) in $p$-Si, on the other hand.

We have confirmed that the radiation hardness of $p$-Si is higher than that for $\mathrm{n}-\mathrm{Si}$ and clarified the reasons for this fact. The radiation hardness is determined by the $n \rightarrow p$ conversion of the conducting matrix in $n$-type silicon and is related, in $p$-type silicon, to the dose, at which the full overlapping of clusters takes place.

\section{References}

1. K.L. Starostin, The temperature dependence of the decreasing rate of electron concentration in $n-G e$ and $\mathrm{n}-\mathrm{Si}$ under the irradiation by fast neutrons // Fizika Tekhnika Poluprovodn. 4 (9), p. 1823-1824 (1970).
2. G.S. Karumidze, Effect of neutron-irradiation temperature on structure-defect formation in silicon grown by the Czochralski method // Fizika Tekhnika Poluprovodn. 24 (11), p. 1973-1977 (1990).

3. A.P. Dolgolenko, P.G. Litovchenko, M.D. Varentsov, G.P. Gaidar, A.P. Litovchenko, The particularities of the formation of radiation defects in silicon with low and high concentrations of oxygen // Scientific Papers of the Institute for Nuclear Research No. 2(15), p. 106-114 (2005).

4. B.R. Gossick, Disordered regions in semiconductors bombarded by fast neutrons // J. Appl. Phys. 30 (8), p. 1214-1218 (1959).

5. A.P. Dolgolenko, I.I. Fishchuk, A-centres build-up kinetics in the conductive matrix of pulled n-type silicon with calculation of their recharges at defect clusters // Phys. status solidi (a) 67 (8), p. 407-411 (1981).

6. P. Kaminski, R. Kozlowski, E. NossarzewskaOrlowska, Formation of electrically active defects in neutron irradiated silicon // Nucl. Instrum. and Meth. Phys. Res. B 186, p. 152-156 (2002); Highresolution photoinduced transient spectroscopy of neutron irradiated bulk silicon // Nucl. Instrum. and Meth. Phys. Res. A 476 (3), p. 639-644 (2002).

7. I. Pintilie, C. Tivarus, L. Pintilie, M. Moll, E. Fretwurst, G. Lindstrom, Thermally stimulated current method applied to highly irradiated silicon diodes // Nucl. Instrum. and Meth. Phys. Res. A 476, p. 652-657 (2002).

8. A.P. Dolgolenko, P.G. Litovchenko, M.D. Varentsov, G.P. Gaidar, A.P. Litovchenko, Particularities of the formation of radiation defects in silicon with low and high concentrations of oxygen // Phys. status solidi (b) 243 (8), p. 1842-1852 (2006).

9. C.P. Ewels, R. Jones, S. Oberg, J. Miro, P. Deak, Shallow thermal donor defects in silicon // Phys. Rev. Lett. 77 (5), p. 865-868 (1996).

10. Y.-H. Lee, N.N. Gerasimenko, J.W. Corbett, EPR study of neutron-irradiated silicon: A positive charge state of the $\langle 100\rangle$ split di-interstitial // Phys. Rev. B 14 (10), p. $4506-4520$ (1976).

11. I.D. Konozenko, A.K. Semenyuk, V.I. Khivrich, Radiation defects created by ${ }^{60}$ Co-rays in $\mathrm{n}$ - and $\mathrm{p}$ type Si of high-purity // Phys. status solidi 35 (2), p. 1043-1052 (1969); Radiation defects in Si of high purity // Radiation Effects 8, p. 121-127 (1971). 